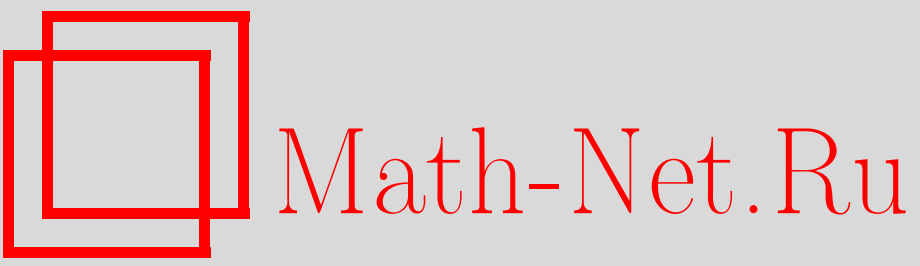

П. А. Вельмисов, Ю. В. Покладова, У. Д. Мизхер, О некоторых начальнокраевых задачах в аэрогидроупругости, Итоги науки и техн. Сер. Соврем. мат. и ее прил. Темат. обз., 2021, том 190, 19-33

DOI: https://doi.org/10.36535/0233-6723-2021-190-19-33

Использование Общероссийского математического портала Math-Net.Ru подразумевает, что вы прочитали и согласны с пользовательским соглашением

http: //www.mathnet.ru/rus/agreement

Параметры загрузки:

IP : 54.174 .149 .18

26 апреля 2023 г., $17: 29: 38$ 


\title{
О НЕКОТОРЫХ НАЧАЛЬНО-КРАЕВЫХ ЗАДАЧАХ В АЭРОГИДРОУПРУГОСТИ
}

\author{
(c) 2021 г. П. А. ВЕЛЬМИСОВ, Ю. В. ПОКЛАДОВА, У. Д. МИЗХЕР
}

\begin{abstract}
АннотАция. Рассматриваются математические модели в задачах о динамике и устойчивости деформируемых элементов конструкций, взаимодействующих с потоком жидкости или газа. Математические модели представляют собой начально-краевые задачи для связанных систем дифференциальных уравнений с частными производными для гидродинамических функций и функций деформаций упругих элементов. Для исследования динамики и устойчивости деформируемых элементов, взаимодействующих с идеальной средой, использовались методы теории функций комплексного переменного, метод Фурье, метод Бубнова-Галеркина и метод функционалов Ляпунова.
\end{abstract}

Ключевые слова: аэрогидроупругость, упругая пластина, деформация, динамика, устойчивость, дифференциальное уравнение с частными производными, метод Бубнова-Галеркина, метод функционалов Ляпунова.

\section{ON SOME INITIAL-BOUNDARY-VALUE PROBLEMS IN AEROHYDROELASTICITY}

\author{
(c) 2021 P. A. VELMisov, YU. V. POKLADOVA, U. J. MIZHER
}

\begin{abstract}
In this problem, we consider problems of the dynamics and stability of deformable structural elements interacting with a fluid or gas flow. Mathematical models considered are initialboundary-value problems for coupled systems of partial differential equations for hydrodynamic functions and deformation functions of elastic elements. To study the dynamics and stability of deformable elements interacting with an ideal medium, we use various methods of the theory of functions of complex variables, the Fourier method, the Bubnov-Galerkin method, and the method of Lyapunov functionals.
\end{abstract}

Keywords and phrases: aerohydroelasticity, elastic plate, deformation, dynamics, stability, partial differential equations, Bubnov-Galerkin method, method of Lyapunov functionals.

AMS Subject Classification: 35Q35, 35Q74

1. Введение. В работе рассматриваются математические модели в задачах о динамике и устойчивости деформируемых элементов конструкций, взаимодействующих с потоком жидкости (газа) при различных режимах обтекания (дозвуковом, сверхзвуковом). В качестве примера предложены математические модели механической системы «трубопровод - датчик давления» и математическая модель, описывающая динамику пластины-полосы в сверхзвуковом потоке газа.

Работа выполнена при поддержке Российского фонда фундаментальных исследований и Правительства Ульяновской области (проект № 18-41-730015). 
Описания датчиков измерительных систем представлены в $[16,19,20,23,24]$. В этих работах приводятся некоторые технические характеристики датчиков и некоторые уравнения, описывающие колебания их упругих элементов. В некоторых публикациях предлагается комплекс технических решений, позволяющий существенно повысить метрологические и эксплуатационные характеристики датчиков. Также в отдельных публикациях рассмотрено влияние нестационарной температуры измеряемой среды на погрешность измерения и предложена методика ее уменьшения, основанная на внесении изменений в конструкцию известных тензорезисторных датчиков давления.

При эксплуатации датчиков давления в авиационных и ракетных двигателях характерной особенностью является воздействие на них нестационарной температуры и повышенных виброускорений, в наибольшей степени проявляющееся в переходных режимах работы двигателя (например, при взлете и посадке аппарата). Такое негативное воздействие приводит к погрешности измерений, а в ряде случаев к разрушению упругого чувствительного элемента датчика. Возникает задача проектирования механической системы «трубопровод - датчик давления». В системе датчик расположен на некотором расстоянии от двигателя и соединен с ним с помощью трубопровода, что позволяет ослабить воздействие высоких температур и виброускорений. Задача состоит в получении уравнений, связывающих закон изменения рабочей среды на входе в трубопровод (на выходе из камеры сгорания двигателя) и деформацию упругого элемента датчика, и предназначенных по величине деформации элемента рассчитать давление в двигателе. Математические модели системы «трубопровод - датчик давления» рассматривались, например, в [4, 7, 9-12,22].

При проектировании аэрогидроупругих систем (конструкций, приборов, устройств, установок и т. д., взаимодействующих с потоком газа или жидкости), важное значение имеет исследование динамики и устойчивости деформируемых элементов, так как воздействие потока может приводить к значениям амплитуды, частоты и скорости колебаний, не позволяющим осуществлять надежную эксплуатацию систем и обеспечивать необходимую надежность и функциональную точность их работы. Среди последних исследований по динамике и устойчивости пластин и оболочек, находящихся в потоке жидкости или газа, следует отметить исследования $[1,2,15,17,21,25,26,28,29]$. Исследованию динамики и устойчивости трубопроводов и их элементов, при протекании внутри них потока жидкости или газа, посвящены, например, работы $[5,14,27,30,31]$. Задачи о сверхзвуковом обтекании возникают, в том числе, при исследовании динамики корпуса ракеты, панели фюзеляжа или его составных частей в случае сверхзвукового режима полета. Среди работ авторов данной статьи по исследованию динамики и устойчивости упругих тел, взаимодействующих с потоком газа, отметим монографии и статьи $[3,8,9,32-34]$.

Построенные в работе модели описываются связанной системой дифференциальных уравнений в частных производных для неизвестных функций - потенциала скорости газа (жидкости), который обтекает конструкцию, и функций деформаций упругих элементов. Для описания механического поведения материала элементов используются следующие модели деформируемого тела: линейные и нелинейные модели упругого тела, учитывающие только поперечную составляющую деформации, и нелинейные модели упругого тела, учитывающие как поперечную, так и продольную составляющие деформации. Численно-аналитическое решение основано на методе Бубнова-Галеркина. Исследование динамической устойчивости осуществлялось на основе построения положительно определенных функционалов типа Ляпунова. Проведен численный эксперимент с целью определения характера колебаний.

2. Математическая модель системы «трубопровод-датчик давления». Если продольный размер трубопровода значительно превосходит его поперечный размер $y_{0}$, то можно считать длину трубопровода бесконечной (см. рис. 1: 1 -трубопровод, 2 -датчик, 3 -рабочая среда, 4 - пластина (упругий элемент)).

2.1. Постановка задачи. Математическая постановка задачи имеет вид:

$$
\begin{gathered}
\phi_{x x}+\phi_{y y}=0, \quad(x, y) \in G=\left\{(x, y): 0<x<+\infty, 0<y<y_{0}\right\}, \\
\phi_{y}(x, 0, t)=\phi_{y}\left(x, y_{0}, t\right)=0, \quad x \in(0,+\infty),
\end{gathered}
$$




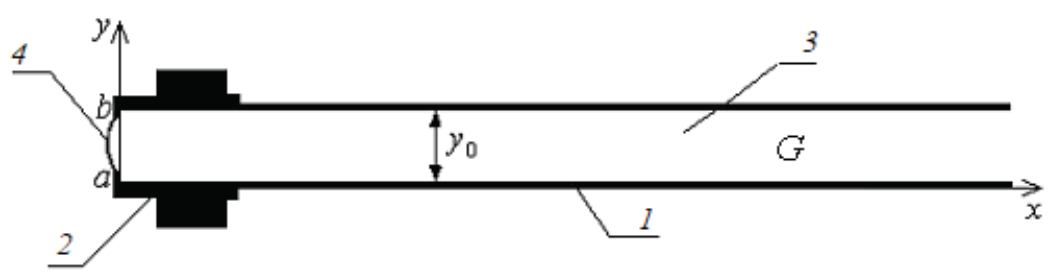

Рис. 1. Бесконечно длинный трубопровод с упругим элементом на торцевой стенке

$$
\begin{gathered}
\phi_{x}(0, y, t)=\dot{w}(y, t), \quad y \in(a, b), \quad 0<a<b<y_{0}, \\
\phi_{x}(0, y, t)=0, \quad y \in(0, a) \cup\left(b, y_{0}\right), \\
\lim _{x \rightarrow+\infty}\left(\phi_{x}^{2}+\phi_{y}^{2}\right)=0 \\
\lim _{x \rightarrow+\infty}\left(\tilde{P}-\rho \phi_{t}\right)=P_{*}(t), \\
w(y, 0)=f_{1}(x), \quad \dot{w}(y, 0)=f_{2}(x), \\
L(w) \equiv M \ddot{w}+D w^{\prime \prime \prime \prime}+N w^{\prime \prime}+\alpha \dot{w}^{\prime \prime \prime \prime}-\delta \ddot{w}^{\prime \prime}+\beta \dot{w}+\gamma w=P_{0}(y, t)-\tilde{P}+\rho \phi_{t}(0, y, t), \quad y \in(a, b) .
\end{gathered}
$$

Здесь $x, y$-декартовы координаты, $t$-время; $\phi(x, y, t)$ - потенциал скорости рабочей среды (жидкости или газа); $w(y, t)$ - прогиб упругого элемента (пластины); $\tilde{P}, \rho$ - давление и плотность рабочей среды в трубопроводе в состоянии покоя; $P_{0}(y, t)$ - распределенная внешняя нагрузка, действующая на упругий элемент; (1)-уравнение Лапласа, описывающее движение рабочей среды в трубопроводе; (2)-(4) - условия непротекания среды через соответствующие границы; условия (5), (6) означают, что на входе в трубопровод (на выходе из двигателя) среда покоится, а закон изменения давления определяется функцией $P_{*}(t) ;(7)$ - начальные условия; (8)уравнение динамики пластины; $a, b$-координаты концов упругого элемента; $M, D$ - погонная масса и изгибная жесткость пластины; $M=\rho_{0} h ; D=E h^{3} /\left(12\left(1-\nu^{2}\right)\right) ; E$ - модуль упругости; $\nu$-коэффициент Пуассона; $h$ - толщина пластины; $\rho_{0}$ - плотность пластины; $N$ - сжимающее (растягивающее) пластину усилие; $\alpha$-коэффициент внутреннего демпфирования; $\beta$ и $\gamma-$ коэффициенты демпфирования и жесткости основания; $\delta$ - коэффициент, учитывающий инерцию поворота сечений; нижние индексы $x, y, t$ обозначают частные производные по $x, y, t$, точкачастную производную по $t$, штрих - частную производную по $y$.

При изменении теплового воздействия на элемент с течением времени растягивающее (сжимающее) усилие $N$ является функцией времени и определяется по формуле

$$
N(t)=N_{0}+\frac{E \alpha_{T}}{1-\nu} \int_{-h / 2}^{h / 2} T(z, t) d z
$$

где $\alpha_{T}$ - температурный коэффициент линейного расширения, $T(z, t)$ - закон изменения температуры по толщине пластины, $N_{0}$ - постоянная составляющая усилия, обусловленная конструктивными особенностями системы.

2.2. Решение аэрогидродинамической задачи методами теории функций комплексного переменного. Считая $t$ параметром, введем в рассмотрение комплексный потенциал $W=f(z, t)=\phi+i \psi$, где $z=x+i y, \psi=\psi(x, y, t)$ - функция тока. Функция $\zeta=\operatorname{ch}(\pi z) / y_{0}$ конформно отображает полуполосу $G$ на верхнюю полуплоскость комплексного переменного $\zeta=\xi+i \eta$ со следующим соответствием точек:

$$
z=\infty \Longleftrightarrow \zeta=\infty, \quad z=0 \Longleftrightarrow \zeta=1, \quad z=i y_{0} \Longleftrightarrow \zeta=-1 .
$$


Упругому элементу (отрезку $[a, b]$ оси $O y)$ в $\zeta$-плоскости соответствует отрезок $\left[\xi_{1}, \xi_{2}\right]$ оси $O \xi$, где

$$
\xi_{1}=\cos \frac{\pi b}{y_{0}}, \quad \xi_{2}=\cos \frac{\pi a}{y_{0}} .
$$

Для аналитической в верхней полуплоскости функции $f_{z}(z(\zeta), t)=\phi_{x}-i \phi_{y}$ имеем согласно (2)-(4) следующие краевые условия:

$$
\begin{gathered}
\operatorname{Im} f_{z}=-\phi_{y}=0, \quad \xi \in(-\infty,-1) \cup(1,+\infty), \\
\operatorname{Re} f_{z}=\phi_{x}= \begin{cases}\dot{\omega}(y(\xi), t), & \xi \in\left(\xi_{1}, \xi_{2}\right), \\
0, & \xi \in\left(-1, \xi_{1}\right) \cup\left(\xi_{2}, 1\right) .\end{cases}
\end{gathered}
$$

Полагая $\zeta=\xi, z=i y$ в формуле $\zeta=\operatorname{ch}(\pi z) / y_{0}$, находим

следовательно,

$$
\xi=\operatorname{ch} \frac{\pi i y}{y_{0}}=\cos \frac{\pi y}{y_{0}}
$$

$$
y=y(\xi)=\frac{y_{0}}{\pi} \arccos \xi, \quad \xi \in(-1,1) .
$$

Таким образом, для аналитической функции $f_{z}(z(\zeta), t)$ имеем смешанную краевую задачу в верхней полуплоскости. Решение этой задачи, ограниченное в точках $\zeta= \pm 1$ и удовлетворяющее условию (5), запишем по формуле Келдыша-Седова (см. [18])

$$
f_{z}(z(\zeta), t)=\frac{R(\zeta)}{\pi i} \int_{\xi_{1}}^{\xi_{2}} \frac{\dot{w}(y(\tau), t) d \tau}{R(\tau)(\tau-\zeta)}
$$

где $R(\zeta)=\sqrt{\zeta^{2}-1}>0$ при $\zeta=\xi>1$. При этом должно быть выполнено условие разрешимости

$$
\int_{\xi_{1}}^{\xi_{2}} \frac{\dot{w}(y(\tau), t)}{R(\tau)} d \tau=0
$$

Учитывая, что $R(\tau)=i \sqrt{1-\tau^{2}}, \tau \in(-1,1)$, с помощью подстановки $\tau=\cos (\pi y) / y_{0}$ представим его в виде

$$
\int_{a}^{b} \dot{w}(y, t) d y=0 .
$$

Условие разрешимости (11) означает равенство нулю потока вектора скорости через границу области $G$, что соответствует модели несжимаемой среды. Далее, поскольку $\zeta=\operatorname{ch}(\pi z) / y_{0}$, имеем

$$
\frac{d \zeta}{d z}=\frac{\pi}{y_{0}} \operatorname{sh} \frac{\pi z}{y_{0}}=\frac{\pi}{y_{0}} \sqrt{\zeta^{2}-1}
$$

Тогда с учетом (10) получаем

$$
W_{\zeta}=f_{z} \cdot \frac{d z}{d \zeta}=\frac{y_{0}}{\pi \sqrt{\zeta^{2}-1}} \cdot f_{z}=-\frac{y_{0}}{\pi^{2}} \int_{\xi_{1}}^{\xi_{2}} \frac{\dot{w}(y(\tau), t)}{\sqrt{1-\tau^{2}}} \frac{d \tau}{\tau-\zeta} .
$$

Интегрируя по Ц полученное выражение, найдем комплексный потенциал

$$
W=\frac{y_{0}}{\pi^{2}} \int_{\xi_{1}}^{\xi_{2}} \frac{\dot{w}(y(\tau), t)}{\sqrt{1-\tau^{2}}} \ln (\tau-\zeta) d \tau+C(t),
$$

где $C(t)$ - произвольная комплексная функция. 
Перейдем в (12) к пределу при $\zeta \rightarrow \xi \in\left(\xi_{1}, \xi_{2}\right)$, при этом $z \rightarrow i y, y \in(a, b)$. Согласно предельным формулам для интеграла с логарифмическим ядром [13] имеем

$$
\phi+i \psi=\frac{y_{0}}{\pi^{2}}\left(\int_{\xi_{1}}^{\xi_{2}} \frac{\dot{w}(y(\tau), t)}{\sqrt{1-\tau^{2}}} \ln |\tau-\xi| d \tau-\pi i \int_{\xi_{1}}^{\xi} \frac{\dot{w}(y(\tau), t)}{\sqrt{1-\tau^{2}}} d \tau\right)+C(t) .
$$

Отсюда, отделяя вещественную часть и дифференцируя ее по $t$, получим

$$
\phi_{t}(0, y, t)=\frac{y_{0}}{\pi^{2}} \int_{\xi_{1}}^{\xi_{2}} \frac{\ddot{w}(y(\tau), t)}{\sqrt{1-\tau^{2}}} \ln |\tau-\xi| d \tau+A(t)
$$

где $\xi=\cos (\pi y) / y_{0}, A(t)=\operatorname{Re} C^{\prime}(t)$.

Подберем произвольную пока функцию $A(t)$ так, чтобы выполнялось условие (6). С этой целью продифференцируем (12) по $t$ и отделим вещественную часть. В результате будем иметь

$$
\phi_{t}=\frac{y_{0}}{\pi^{2}} \int_{\xi_{1}}^{\xi_{2}} \frac{\ddot{w}(y(\tau), t)}{\sqrt{1-\tau^{2}}} \ln |\tau-\zeta| d \tau+A(t),
$$

или, с учетом условия (11),

$$
\phi_{t}=\frac{y_{0}}{\pi^{2}} \int_{\xi_{1}}^{\xi_{2}} \frac{\ddot{w}(y(\tau), t)}{\sqrt{1-\tau^{2}}} \ln \left|\frac{\tau}{\zeta}-1\right| d \tau+A(t) .
$$

При $\zeta \rightarrow \infty(x \rightarrow+\infty)$ из (14) и (6) получаем $A(t)=\frac{\tilde{P}-P_{*}(t)}{\rho}$. Формулу (13), сделав в интегральном слагаемом подстановку $\tau=\cos \frac{\pi s}{y_{0}}$, можно теперь записать в виде

$$
\phi_{t}(0, y, t)=\frac{1}{\pi} \int_{a}^{b} \ddot{w}(s, t) \ln \left|\cos \frac{\pi s}{y_{0}}-\cos \frac{\pi y}{y_{0}}\right| d s+\frac{\tilde{P}-P_{*}(t)}{\rho} .
$$

Подставляя (15) в (8), получим уравнение, связывающее закон изменения давления $P_{*}(t)$ на входе в трубопровод $\left(x_{0}=+\infty\right)$ и функцию прогиба (деформацию) $w(y, t)$ упругого элемента датчика, расположенного в сечении $x=0$

$$
L(w)=P_{0}(y, t)-P_{*}(t)+\frac{\rho}{\pi} \int_{a}^{b} \ddot{w}(s, t) \ln \left|\cos \frac{\pi s}{y_{0}}-\cos \frac{\pi y}{y_{0}}\right| d s .
$$

2.3. Решение аэрогидродинамической задачи методом Фурье. Уравнение для функции деформации упругого элемента можно получить на основе метода Фурье.

Потенциал скорости рабочей среды будем искать в виде функции, являющейся решением уравнения Лапласа (1) и удовлетворяющей условиям (2), (5):

$$
\phi(x, y, t)=\phi_{0}(t)+\sum_{n=1}^{\infty} \phi_{n}(t) \cos \left(\lambda_{n} y\right) e^{-\lambda_{n} x} .
$$

Здесь $\lambda_{n}=\frac{n \pi}{y_{0}}$, а $\phi_{0}(t), \phi_{n}(t)$ произвольные функции. Удовлетворяя условию $(6)$, получим

$$
\dot{\phi}_{0}(t)=\frac{\tilde{P}-P_{*}(t)}{\rho} .
$$

Подставляя функцию (17) в условия (3), (4), получим

$$
-\sum_{n=1}^{\infty} \phi_{n}(t) \lambda_{n} \cos \left(\lambda_{n} y\right)= \begin{cases}\dot{w}(y, t), & y \in(a, b), \\ 0, & y \in(0, a) \cup\left(b, y_{0}\right) .\end{cases}
$$


Проецируя полученное выражение на систему $\left\{\cos \left(\lambda_{n} y\right)\right\}_{0}^{\infty}$, будем иметь

$$
\phi_{n}(t)=-\frac{2}{\lambda_{n} y_{0}} \int_{a}^{b} \dot{w}(y, t) \cos \left(\lambda_{n} y\right) d y, \quad n=1,2, \ldots,
$$

и условие разрешимости

$$
\int_{a}^{b} \dot{w}(y, t) d y=0
$$

Подставляя найденные выражения для $\dot{\phi_{0}}(t), \dot{\phi}_{n}(t)$ в правую часть уравнения $(8)$, получим уравнение, связывающее деформацию упругого элемента датчика и давление на входе в трубопровод:

$$
L(w)=P_{0}(y, t)-P_{*}(t)-\frac{2 \rho}{y_{0}} \sum_{n=1}^{\infty} \frac{\cos \left(\lambda_{n} y\right)}{\lambda_{n}} \int_{a}^{b} \ddot{w}(y, t) \cos \left(\lambda_{n} y\right) d y, \quad \lambda_{n}=\frac{n \pi}{y_{0}} .
$$

2.4. Случай резонанса. Пусть $a=0, b=y_{0}$. Рассмотрим подвижное жесткое защемление концов упругого элемента, когда граничные условия имеют вид $w^{\prime}(0, t)=w^{\prime}\left(y_{0}, t\right)=0, w^{\prime \prime \prime}(0, t)=$ $w^{\prime \prime \prime}\left(y_{0}, t\right)=0$. Полагая в $(18)$

$$
w(y, t)=\sum_{n=0}^{\infty} w_{n}(t) \cos \left(\lambda_{n} y\right), \quad P_{0}(y, t)=\sum_{n=0}^{\infty} P_{0 n}(t) \cos \left(\lambda_{n} y\right)
$$

для $w_{n}(t)(n=0,1, \ldots)$, получим не связанные между собой уравнения

$$
\left\{\begin{array}{l}
M \ddot{w}_{0}+\beta \dot{w}_{0}+\gamma w_{0}=P_{00}(t)-P_{*}(t), \\
\left(M+\rho / \lambda_{n}\right) \ddot{w}_{n}+\left(\alpha \lambda_{n}^{4}+\beta\right) \dot{w}_{n}+\left(D \lambda_{n}^{4}-N \lambda_{n}^{2}+\gamma\right) w_{n}=P_{0 n}(t) .
\end{array}\right.
$$

В качестве примера рассмотрим периодическое изменение давления с течением времени $P_{00}(t)-$ $P_{*}(t)=B_{0} \sin \omega_{0} t, P_{0 n}(t)=B_{n} \sin \omega_{n} t$. Полагая $w_{0}(t)=A_{0} \sin \omega_{0} t, w_{n}(t)=A_{n} \sin \omega_{n} t$, из (19) получим

$$
\left\{\begin{array}{l}
-M A_{0} \omega_{0}^{2}+\gamma A_{0}=B_{0}, \\
-\left(M+\rho / \lambda_{n}\right) A_{n} \omega_{n}^{2}+\left(D \lambda_{n}^{4}-N \lambda_{n}^{2}+\gamma\right) A_{n}=B_{n},
\end{array}\right.
$$

откуда следует наличие резонансных частот по каждой из гармоник

$$
\omega_{0}=\sqrt{\frac{\gamma}{M}}, \quad \omega_{n}=\sqrt{\frac{D \lambda_{n}^{4}-N \lambda_{n}^{2}+\gamma}{M+\rho / \lambda_{n}}} .
$$

При этом для существования резонансных частот необходимо выполнение условий

$$
\gamma>0, \quad N<D \lambda_{n}^{2}+\frac{\gamma}{\lambda_{n}^{2}}
$$

2.5. Нелинейные математические модели системы «трубопровод-датчик давления». Постановка задачи (1)-(8) соответствует линейной теории аэрогидроупругости, когда динамика жидкости (газа), а также динамика чувствительного элемента датчика описываются линейными уравнениями.

Рассмотрим нелинейную одностепенную модель, которая определяется приведенными выше уравнениями (1)-(7), при этом в уравнении (8), описывающем динамику пластины, интегро-дифференциальный оператор $L(w)$ заменяется следующим

$L(w) \equiv M \ddot{w}+\left[\frac{D w^{\prime \prime}}{\left[1+\left(w^{\prime}\right)^{2}\right]^{3 / 2}}\right]^{\prime \prime}+N w^{\prime \prime}+\alpha \dot{w}^{\prime \prime \prime \prime}-\delta \ddot{w}^{\prime \prime}+\beta \dot{w}+\gamma w+\mu w^{3}-\theta w^{\prime \prime}\left[\int_{a}^{b} \sqrt{1+\left(w^{\prime}\right)^{2}} d y+a-b\right]$. 
Слагаемое

$$
\theta w^{\prime \prime}\left[\int_{a}^{b} \sqrt{1+\left(w^{\prime}\right)^{2}} d y+a-b\right]
$$

учитывает нелинейную продольную силу, возникающую при деформации элемента, слагаемое

$$
\left[\frac{D w^{\prime \prime}}{\left[1+\left(w^{\prime}\right)^{2}\right]^{3 / 2}}\right]^{\prime \prime}
$$

учитывает нелинейность изгибающего момента.

Полагая прогибы малыми, заменим $\left[1+\left(w^{\prime}\right)^{2}\right]^{-3 / 2}$ и $\sqrt{1+\left(w^{\prime}\right)^{2}}$ на $\left[1-\frac{3}{2}\left(w^{\prime}\right)^{2}\right]$ и $\left[1+\frac{1}{2}\left(w^{\prime}\right)^{2}\right]$ соответственно. Получим

$$
\begin{array}{rl}
L(w) \equiv M \ddot{w}+D w^{\prime \prime \prime \prime}-\frac{3}{2} D w^{\prime \prime \prime \prime}\left(w^{\prime}\right)^{2}-9 & D w^{\prime} w^{\prime \prime} w^{\prime \prime \prime}-3 D\left(w^{\prime \prime}\right)^{3}+N w^{\prime \prime}+ \\
& +\alpha \dot{w}^{\prime \prime \prime \prime}-\delta \ddot{w}^{\prime \prime}+\beta \dot{w}+\gamma w+\mu w^{3}-\frac{1}{2} \theta w^{\prime \prime} \int_{a}^{b}\left(w^{\prime}\right)^{2} d y .
\end{array}
$$

Рассмотрим также нелинейную модель системы «трубопровод-датчик давления», учитывающую как поперечную, так и продольную деформации упругого элемента датчика. Уравнение (8), описывающее динамику упругого элемента, заменяется системой двух уравнений (двухстепенная модель):

$$
\left\{\begin{aligned}
-E F\left[u^{\prime}+\frac{1}{2}\left(w^{\prime}\right)^{2}\right]^{\prime}+M \ddot{u} & +\alpha_{*} \dot{u}^{\prime \prime}=0 \\
-E F\left[w^{\prime}\left(u^{\prime}+\frac{1}{2}\left(w^{\prime}\right)^{2}\right)\right]^{\prime} & +\left[\frac{D w^{\prime \prime}}{\left[1+\left(w^{\prime}\right)^{2}\right]^{3 / 2}}\right]^{\prime \prime}+M \ddot{w}+\alpha \dot{w}^{\prime \prime \prime \prime}-\delta \ddot{w}^{\prime \prime}+ \\
& +\beta \dot{w}+N w^{\prime \prime}+\gamma w=P_{0}(y, t)-\tilde{P}+\rho \phi_{t}(0, y, t), \quad y \in(a, b) .
\end{aligned}\right.
$$

Здесь $u(y, t), w(y, t)$ - продольная и поперечная деформации упругого элемента; $E$ - модуль упругости; $\nu$ - коэффициент Пуассона; $h$ - толщина пластинки;

$$
M=\rho_{0} h ; \quad F=\frac{h}{1-\nu^{2}} ; \quad D=\frac{E h^{3}}{12\left(1-\nu^{2}\right)} .
$$

В предположении, что $w^{\prime}$ мало, производилась замена

$$
\frac{1}{\left[1+\left(w^{\prime}\right)^{2}\right]^{3 / 2}} \rightarrow\left[1-\frac{3}{2}\left(w^{\prime}\right)^{2}\right] .
$$

Для нелинейной одностепенной модели (1)-(7), (20) решение $w(y, t)$ уравнения (16) представляется в виде

$$
w(y, t)=\sum_{k=1}^{n} w_{k}(t) g_{k}(y),
$$

где $\left\{g_{k}(y)\right\}_{k=1}^{\infty}$ - полная на $[a, b]$ система базисных функций, удовлетворяющих граничным условиям, соответствующим условиям закрепления пластины.

Из условия ортогональности невязки уравнения (16) к системе базисных функций $\left\{g_{k}(y)\right\}_{k=1}^{n}$ получим систему из $n$ обыкновенных дифференциальных уравнений для $w_{k}(t)$.

Для нелинейной двухстепенной модели (1)-(7), (21), учитывающей как продольную, так и поперечную деформации, согласно методу Галеркина, искомые функции деформации $u(y, t), w(y, t)$ ищем в виде

$$
u(y, t)=\sum_{k=1}^{n} u_{k}(t) h_{k}(y), \quad w(y, t)=\sum_{k=1}^{n} w_{k}(t) g_{k}(y),
$$

где $\left\{h_{k}(y)\right\}_{k=1}^{\infty},\left\{g_{k}(y)\right\}_{k=1}^{\infty}$ - полные на $[a, b]$ системы базисных функций, удовлетворяющих граничным условиям, соответствующим условиям закрепления пластины. 


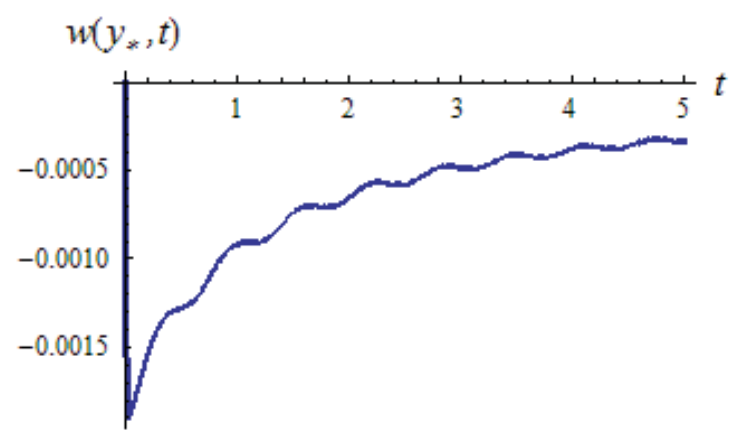

Рис. 2. График деформации $w\left(y_{*}, t\right)$ для линейной модели

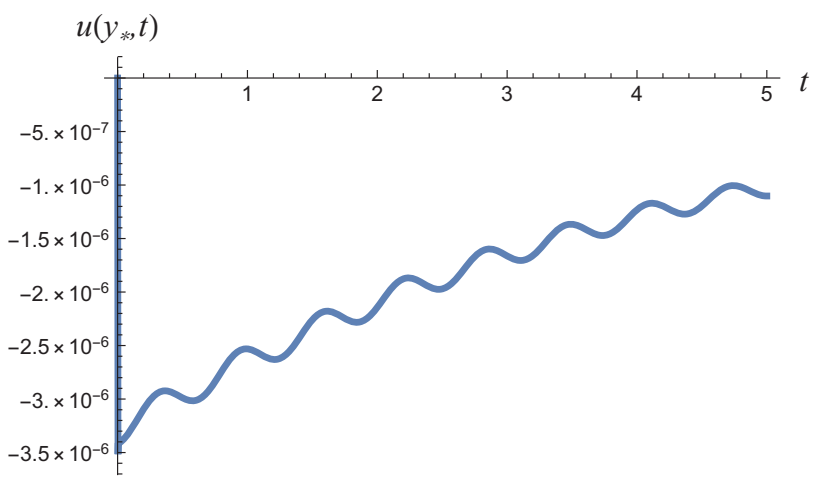

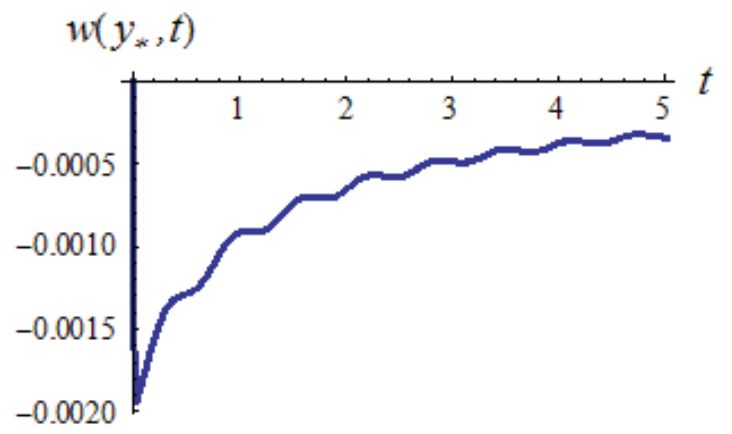

Рис. 3. График деформации $w\left(y_{*}, t\right)$ для одностепенной нелинейной модели

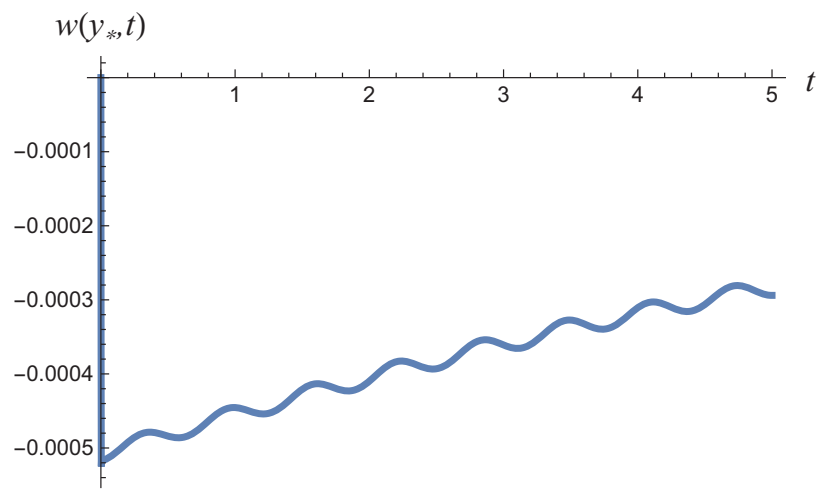

Рис. 4. Графики деформации $u\left(y_{*}, t\right), w\left(y_{*}, t\right)$ для двухстепенной нелинейной модели

Из условия ортогональности невязки первого уравнения системы (21) к системе базисных функций $\left\{h_{k}(y)\right\}_{k=1}^{n}$, второго уравнения системы (21) к системе базисных функций $\left\{g_{k}(y)\right\}_{k=1}^{n}$ получим систему из $2 n$ обыкновенных дифференциальных уравнений для $u_{k}(t), w_{k}(t)$.

Для всех рассмотренных моделей задача Коши для систем обыкновенных дифференциальных уравнений решается с помощью системы Mathematica 12.0. Проведено численное моделирование на ЭВМ динамики упругого элемента датчика в зависимости от закона изменения давления в двигателе. Исследовалась деформация упругого элемента для различных параметров механической системы.

2.6. Численный эксперимент. Проведем исследование динамики упругого элемента датчика с учетом теплового воздействия $(N(t)$ - функция времени). В случае шарнирного закрепления концов упругого элемента искомые функции деформации $u(x, t), w(x, t)$ ищем в виде

$$
u(x, t)=\sum_{k=1}^{n} u_{k}(t) \sin \left(\lambda_{k}(x-a)\right), \quad w(x, t)=\sum_{k=1}^{n} w_{k}(t) \sin \left(\lambda_{k}(x-a)\right), \quad \lambda_{k}=\frac{\pi k}{b-a},
$$

где $\left\{\sin \left(\lambda_{k}(x-a)\right)\right\}_{k=1}^{\infty}-$ полная на $[a, b]$ система базисных функций, удовлетворяющих шарнирному закреплению пластины. Рабочая среда-вода $\left(\rho=10^{3}\right)$, пластина изготовлена из стали $\left(E=2 \cdot 10^{11}, \rho_{0}=7,8 \cdot 10^{3}, h=4 \cdot 10^{-4}\right)$. Все значения приведены в единицах СИ.

Для значений параметров $n=2, y_{0}=0,02, a=0, b=0,02, M=3,12, D=1,1722, \alpha=0,01$, $\alpha_{*}=0,01, \beta=0,3, \mu=0, \theta=0, \delta=0, w(y, 0)=0, \dot{w}(y, 0)=0,5, P_{0}(y, t)=0, P_{*}(t)=10^{5}(20+$ $\cos 10 t), N(t)=1000-2500(10+20 t)$ получены графики функций $u(y, t), w(y, t)$ в точке $y_{*}=(a+b) / 2,5$ (рис. $\left.2-4\right)$. 


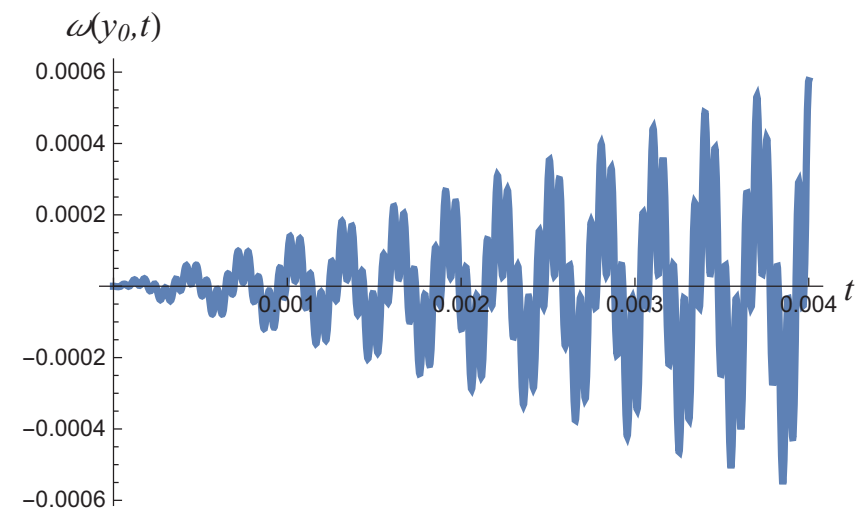

Рис. 5. График деформации $w\left(y_{*}, t\right)$ в случае резонанса

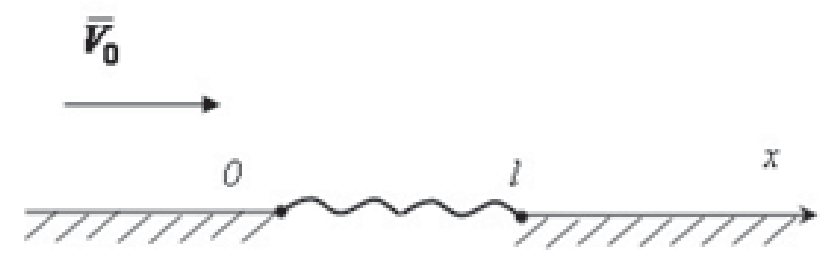

Рис. 6. Пластина, обтекаемая сверхзвуковым потоком газа

Деформация в случае двухстепенной нелинейной модели значительно меньше, чем в линейной и одностепенной нелинейной моделях. Следовательно, учет продольной составляющей деформации имеет существенное значение при исследовании динамики упругого элемента.

Аналогичный численный эксперимент проводился для модели с трубопроводом конечной длины. В случае, когда продольный размер трубопровода значительно превосходит его поперечный размер, графики деформаций для моделей с трубопроводом конечной длины и бесконечно длинным трубопроводом мало отличаются. Поэтому для расчетов систем с трубопроводами достаточной длины можно использовать модель с бесконечно длинным трубопроводом.

Приведем пример численного эксперимента для случая резонанса. Рассмотрим жесткое подвижное закрепление концов упругого элемента. Рабочая среда - вода $\left(\rho=10^{3}\right)$, пластина изготовлена из алюминия $(M=3,12, D=1,1722)$. Все значения приведены в единицах СИ. Для значений параметров $n=2, y_{0}=0,02, a=0, b=y_{0}, \alpha=\beta=0, \gamma=0, w(y, 0)=0, \dot{w}(y, 0)=0$,

$$
\begin{gathered}
P_{0}(y, t)=10^{3} \sin \left(\sqrt{\frac{\gamma}{M}} t\right)+10^{5} \sum_{n=1}^{2} \sin \left(\sqrt{\frac{D \lambda_{n}^{4}-N \lambda_{n}^{2}+\gamma}{M+\frac{\rho}{\lambda_{n}}}} t\right) \cos \left(\lambda_{n} y\right), \\
P_{*}(t)=9 \cdot 10^{2} \sin \left(\sqrt{\frac{\gamma}{M}} t\right)
\end{gathered}
$$

с помощью системы Mathematica 12.0 получен график функции $w(y, t)$ в точке $y_{*}=\frac{a+b}{2,5}$ (рис. 5 ).

3. Математическая модель пластины-полосы в сверхзвуковом потоке газа. Рассмотрим плоскую задачу о динамике упругого элемента (пластины) некоторой конструкции при одностороннем обтекании сверхзвуковым потоком газа в направлении оси $O x$ со скоростью $V_{0}>a_{0}$, где $a_{0}$-скорость звука (рис. 6). Предполагается, что число Маха $M_{0}=V_{0} / a_{0}>\sqrt{2}$. Пластина в недеформированном состоянии занимает положение $0<x<l$ оси $O x$. 
3.1. Постановка задачи. Математическая формулировка задачи имеет вид:

$$
\begin{aligned}
& \phi_{t t}+2 V_{0} \phi_{x t}+V_{0}^{2} \phi_{x x}=a_{0}^{2}\left(\phi_{x x}+\phi_{y y}\right), \\
& \phi_{y}(x, 0, t)= \begin{cases}0, & x \in(-\infty, 0) \cup(l,+\infty), \\
\dot{w}+V_{0} w^{\prime}, & x \in(0, l),\end{cases} \\
& \phi(0, y, t)=\phi_{x}(0, y, t)=0, \quad w(0, t)=0, \\
& \phi(x, y, 0)=\phi_{t}(x, y, 0)=0, \\
& \phi(x, y, t) \rightarrow 0, \quad y \rightarrow \infty, \\
& w(x, 0)=\dot{w}(x, 0)=0, \\
& M \ddot{w}+D w^{\prime \prime \prime \prime}+N w^{\prime \prime}+\alpha \dot{w}^{\prime \prime \prime \prime}+\beta \dot{w}+\gamma w+f(x, w)=p^{-}-p^{+}+\rho\left(\phi_{t}(x, 0, t)+V_{0} \phi_{x}(x, 0, t)\right) .
\end{aligned}
$$

Здесь $x, y$ - декартовы координаты, $t$-время; $\phi(x, y, t)$ - потенциал скорости среды; $w(x, t)-$ прогиб упругого элемента; (22) - уравнение, описывающее течение газа над пластиной в модели идеальной сжимаемой среды; (23) - условия непротекания; (24) - условия отсутствия возмущений перед пластиной; (25) - условия отсутствия возмущений в начальный момент времени над пластиной; (26) - условие затухания возмущений вдали от пластины; (27) - начальные условия; (28) - уравнение, описывающее динамику упругого элемента с учетом воздействия на него сверхзвукового потока газа сверху; $M, D$ - погонная масса и изгибная жесткость пластины; $M=\rho_{0} h$; $D=E h^{3} /\left(12\left(1-\nu^{2}\right)\right) ; E$ - модуль упругости; $\nu$ - коэффициент Пуассона; $h$ - толщина пластины; $\rho_{0}$ - плотность пластины; $\rho, p^{+}$- плотность и давление в невозмущенном однородном потоке; $p^{-}$- давление среды под пластиной; $N$ - сжимающее (растягивающее) пластину усилие; $\alpha-$ коэффициент внутреннего демпфирования; $\beta$ и $\gamma$-коэффициенты демпфирования и жесткости основания; нижние индексы $x, y, t$ обозначают частные производные по $x, y, t$, точка - частную производную по $t$, штрих - частную производную по $y$.

3.2. Решение аэрогидродинамической задачи операционным методом. Для решения задачи применяется операционный метод, позволяющий получить дифференциальное уравнение в частных производных, которое описывает динамику упругой пластины с учетом аэрогидродинамического воздействия на нее и содержит лишь функцию деформации. Суть метода заключается в применении преобразования Лапласа к уравнению (22), условиям (23), (24) по переменным $x$ и $t$ (см., например, [6]). В результате получим изображение для слагаемого $\rho\left(\phi_{t}(x, 0, t)+V_{0} \phi_{x}(x, 0, t)\right)$ из правой части уравнения (28). Для этого изображения при некоторых условиях удается выразить оригинал через линейную комбинацию функций $\dot{w}, w^{\prime}$. После нахождения оригинала получим дифференциальное уравнение в частных производных, которое описывает динамику упругой пластины с учетом аэрогидродинамического воздействия на нее и содержит лишь функцию деформации:

$$
M \ddot{w}+D w^{\prime \prime \prime \prime}+N w^{\prime \prime}+\alpha \dot{w}^{\prime \prime \prime \prime}+\beta \dot{w}+\gamma w+f(x, w)=p^{-}-p^{+}-\frac{\rho V_{0}}{\sqrt{M_{0}^{2}-1}}\left(V_{0} w^{\prime}+\frac{M_{0}^{2}-2}{M_{0}^{2}-1} \dot{w}\right) .
$$

При $M_{0} \rightarrow \infty$ уравнение (29) динамики упругого элемента (пластины) принимает вид

$$
M \ddot{w}+D w^{\prime \prime \prime \prime}+N w^{\prime \prime}+\alpha \dot{w}^{\prime \prime \prime \prime}+\beta \dot{w}+\gamma w+f(x, w)=p^{-}-p^{+}-\rho a_{0}\left(V_{0} w^{\prime}+\dot{w}\right) .
$$

Рассмотрим также нелинейную модель, учитывающую как поперечную, так и продольную деформации упругого элемента. Уравнение (29), описывающее динамику пластины, заменяется системой двух уравнений:

$$
\left\{\begin{aligned}
-E F\left[u^{\prime}+\frac{1}{2}\left(w^{\prime}\right)^{2}\right]^{\prime}+M \ddot{u}+\alpha_{*} \dot{u}^{\prime \prime}+f(x, t, u, w, \dot{u}, \dot{w})=0, & \\
-E F\left[w^{\prime}\left(u^{\prime}+\frac{1}{2}\left(w^{\prime}\right)^{2}\right)\right]^{\prime} & +D w^{\prime \prime \prime \prime}+M \ddot{w}+\alpha \dot{w}^{\prime \prime \prime \prime}+ \\
& +g(x, t, u, w, \dot{u}, \dot{w})=p^{-}-p^{+}-\frac{\rho V_{0}}{\sqrt{M_{0}^{2}-1}}\left(V_{0} w^{\prime}+\frac{M_{0}^{2}-2}{M_{0}^{2}-1} \dot{w}\right) .
\end{aligned}\right.
$$


Здесь $u(x, t), w(x, t)$ - продольная и поперечная деформации упругого элемента; $E$ - модуль упругости; $\nu$ - коэффициент Пуассона; $h$ - толщина пластинки; $M=\rho_{0} h$;

$$
F=\frac{h}{1-\nu^{2}} ; \quad D=\frac{E h^{3}}{12\left(1-\nu^{2}\right)} ;
$$

$f(x, t, u, w, \dot{u}, \dot{w}), g(x, t, u, w, \dot{u}, \dot{w})$ - функции, описывающие некоторые внешние воздействия на упругий элемент. Также необходимо задать начальные условия:

$$
u(x, 0)=f_{1}(x), \quad \dot{u}(x, 0)=f_{2}(x), \quad w(x, 0)=f_{3}(x), \quad \dot{w}(x, 0)=f_{4}(x) .
$$

3.3. Исследование динамической устойчивости. Рассмотрим однородное уравнение, соответствующее уравнению (30)

$$
M \ddot{w}+D w^{\prime \prime \prime \prime}+N w^{\prime \prime}+\alpha \dot{w}^{\prime \prime \prime \prime}+\beta \dot{w}+\gamma w+f(x, w)+\rho a_{0}\left(V_{0} w^{\prime}+\dot{w}\right)=0 .
$$

Здесь $f(x, w)$ - нелинейное воздействие, например, реакция основания. Согласно физическому смыслу задачи параметры $M, D, \rho, a_{0}, V_{0}$ положительны, параметры $\alpha, \beta, \gamma$ неотрицательны.

Умножая уравнение $(32)$ на $(\dot{w}+\theta w)$ и проводя ряд преобразований, получим

$$
\begin{aligned}
& \frac{1}{2} \frac{\partial}{\partial t}\left(M \dot{w}^{2}+2 M \theta \dot{w} w+D w^{\prime \prime 2}-N w^{\prime 2}+\alpha \theta w^{\prime \prime 2}+\beta \theta w^{2}+\gamma w^{2}+2 \int_{0}^{w} f(x, t, z) d z+\rho a_{0} \theta w^{2}\right)+ \\
& +\frac{\partial}{\partial x}\left(-Q_{x}(\dot{w}+\theta w)-M_{x}\left(\dot{w}^{\prime}+\theta w^{\prime}\right)+N w^{\prime} \dot{w}+N \theta w^{\prime} w+\frac{1}{2} \rho a_{0} V_{0} \theta w^{2}\right)+ \\
& +\left(D \theta w^{\prime \prime 2}-N \theta w^{\prime 2}+\alpha \dot{w}^{\prime \prime 2}+\left(\beta-M \theta+\rho a_{0}\right) \dot{w}^{2}+\gamma \theta w^{2}+\theta w f(x, w)+\rho a_{0} V_{0} w^{\prime} \dot{w}\right)=0 .
\end{aligned}
$$

Здесь $M_{x}=D w^{\prime \prime}+\alpha \dot{w}^{\prime \prime}-$ изгибающий момент, $Q_{x}=-\left(D w^{\prime \prime}+\alpha \dot{w}^{\prime \prime}\right)^{\prime}-$ перерезывающая сила. Введем в рассмотрение функционал

$$
\begin{aligned}
J(t)=\frac{1}{2} \int_{0}^{l}\left(M \dot{w}^{2}+2 M \theta \dot{w} w+(D+\alpha \theta)\right. & w^{\prime \prime 2}-N w^{\prime 2}+ \\
& \left.+\left(\beta \theta+\gamma+\rho a_{0} \theta\right) w^{2}+2 \int_{0}^{w} f(x, z) d z\right) d x .
\end{aligned}
$$

Предположим, что концы пластины закреплены следующим образом:

\begin{tabular}{|c|c|c|c|c|}
\hline & 1 & 2 & 3 & 4 \\
\hline$x=0:$ & Ж & Ж & $\amalg$ & $\amalg$ \\
\hline$x=l:$ & Ж & $\amalg$ & Ж & $\amalg$ \\
\hline
\end{tabular}

Здесь введены обозначения: «ж»- жесткое защемление $\left(w=0, w^{\prime}=0\right)$; «ш»- шарнирное закрепление $\left(w=0, M_{x}=0\right)$. Согласно (33) для указанных способов закрепления (35) имеет место равенство

$$
\frac{d J}{d t}=-\int_{0}^{l}\left(D \theta w^{\prime \prime 2}-N \theta w^{\prime 2}+\alpha \dot{w}^{\prime \prime 2}+\left(\beta+\rho a_{0}-M \theta\right) \dot{w}^{2}+\gamma \theta w^{2}+\theta w f(x, w)+\rho a_{0} V_{0} w^{\prime} \dot{w}\right) d x .
$$

Согласно (34), (35) и неравенству Коши-Буняковского справедлива оценка

$$
J(t) \geqslant \frac{1}{2} \int_{0}^{l}\left(M \dot{w}^{2}+2 M \theta w \dot{w}+\left(\frac{2}{l^{2}}\left(\frac{2}{l^{2}}(D+\alpha \theta)-N\right)+\gamma+\theta \beta+\theta \rho a_{0}\right) w^{2}\right) d x .
$$

При этом сделаны предположения

$$
N<\frac{2}{l^{2}}(D+\alpha \theta)+\frac{l^{2}}{2}\left(\gamma+\theta \beta+\theta \rho a_{0}-M \theta^{2}\right), \quad \int_{0}^{w} f(x, z) d z \geqslant 0 .
$$


Аналогично, используя неравенство Коши-Буняковского в (36), получим

$$
\frac{d J}{d t} \leqslant-\int_{0}^{l}\left[\left(\frac{2}{l^{2}} D-N\right) \theta w^{2}+\left(\frac{4}{l^{4}} \alpha+\beta+\rho a_{0}-M \theta\right) \dot{w}^{2}+\rho a_{0} V_{0} w^{\prime} \dot{w}+\gamma \theta w^{2}+\theta w f(x, w)\right] d x .
$$

Полученное неравенство справедливо при условиях:

$$
\theta>0, \quad w f(x, w) \geqslant 0, \quad N \leqslant \frac{2}{l^{2}} D-\frac{\left(\rho a_{0} V_{0}\right)^{2}}{4 \theta\left(\beta+\rho a_{0}-M \theta+\frac{4}{l^{4}} \alpha\right)}, \quad \beta+\rho a_{0}-M \theta+\frac{4}{l^{4}} \alpha>0 .
$$

Таким образом, если выполнены условия

$$
\begin{gathered}
0<\theta<\frac{\beta+\rho a_{0}+\frac{4}{l^{4}} \alpha}{M}, \quad w f(x, w) \geqslant 0, \quad \int_{0}^{w} f(x, z) d z \geqslant 0, \\
N \leqslant \frac{2}{l^{2}} D-\frac{\left(\rho a_{0} V_{0}\right)^{2}}{4 \theta\left(\beta+\rho a_{0}-M \theta+\frac{4}{l^{4}} \alpha\right)} .
\end{gathered}
$$

то $J(t) \geqslant 0, \dot{J}(t) \leqslant 0, J(t) \leqslant J(0)$. Тогда имеет место неравенство, содержащее в левой части под знаком интеграла положительно определенную квадратичную форму для $w, \dot{w}$

$$
\begin{aligned}
& \frac{1}{2} \int_{0}^{l}\left(M \dot{w}^{2}+2 M \theta w \dot{w}+\left(\frac{2}{l^{2}}\left(\frac{2}{l^{2}}(D+\alpha \theta)-N\right)+\gamma+\theta \beta+\theta \rho a_{0}\right) w^{2}\right) d x \leqslant \\
& \leqslant \frac{1}{2} \int_{0}^{l}\left(M \dot{w}^{2}(x, 0)+2 M \theta \dot{w}(x, 0) w(x, 0)+(D+\alpha \theta) w^{\prime \prime 2}(x, 0)-N w^{\prime 2}(x, 0)\right) d x+ \\
& +\frac{1}{2} \int_{0}^{l}\left[\left(\beta \theta+\gamma+\rho a_{0} \theta\right) w^{2}(x, 0)+2 \int_{0}^{w(x, 0)} f(x, z) d z\right] d x .
\end{aligned}
$$

Из данного неравенства вытекает следующая теорема.

Теорема 1. Пусть выполнены условия (35), (37). Тогда имеет место устойчивость в среднем на $[0, l]$ для $w(x, t), \dot{w}(x, t)$ относительно начальных данных $w(x, 0), \dot{w}(x, 0), w^{\prime}(x, 0), w^{\prime \prime}(x, 0)$.

Рассмотрим условия (37). Одно из них накладывает ограничение на значение сжимающего усилия $N$ и скорость потока $V_{0}$. Область устойчивости на плоскости $\left(V_{0}, N\right)$ ограничена семейством парабол с параметром $\theta$

$$
N \leqslant \frac{2}{l^{2}} D-B(\theta) V_{0}^{2}
$$

где $B(\theta)$ - положительная величина.

Укажем пример функции $f(x, w)$, удовлетворяющей условиям $(37)$,

$$
f(x, w)=\sum_{k=1}^{n} f_{k}(x) w^{2 k+1}, \quad f_{k}(x) \geqslant 0, \quad x \in[0, l] .
$$

3.4. Численный эксперимент. Рассмотрим случай шарнирного закрепления концов упругого элемента. Для нелинейной модели $(22)-(27),(31)$, учитывающей как продольную, так и поперечную деформации, согласно методу Галеркина, искомые функции деформации $u(x, t), w(x, t)$ ищем в виде

$$
u(x, t)=\sum_{k=1}^{n} u_{k}(t) \sin \left(\lambda_{k} x\right), \quad w(x, t)=\sum_{k=1}^{n} w_{k}(t) \sin \left(\lambda_{k} x\right),
$$

где $\left\{\sin \left(\lambda_{k} x\right)\right\}_{k=1}^{\infty}-$ полная на $[0, l]$ система базисных функций, удовлетворяющих шарнирному закреплению пластины. 

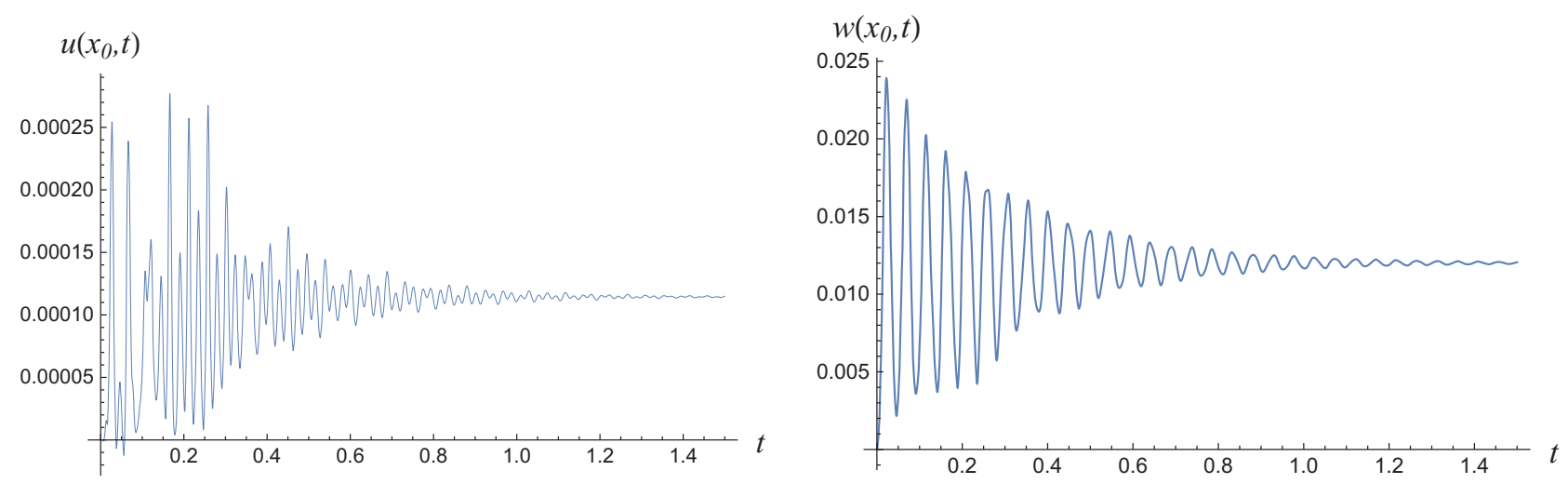

Рис. 7. Графики деформации $u(y, t), w(y, t)$ для нелинейной модели в точке $x=0,8$

Из условия ортогональности невязок первого, второго уравнений системы (31) и начальных условий к системе $\left\{\sin \left(\lambda_{k} x\right)\right\}_{k=1}^{n}$ получим задачу Коши для $u_{k}(t), w_{k}(t)$. Задача Коши решается с помощью системы Mathematica 12.0.

Будем считать, что упругий элемент изготовлен из алюминия $\left(E=2 \cdot 10^{11}\right.$ - модуль упругости; $\rho_{p l}=7,8 \cdot 10^{3}$ - плотность; $h_{p l}=0,005$ - толщина пластины); обтекается сверхзвуковым потоком воздуха. Скорость набегающего потока $V_{0}=600$; скорость звука $a_{0}=340$; плотность воздуха $\rho=1,3 ;$ число Маха $M_{0}=1,765$. Другие параметры механической системы: $l=2 ; M=39$ (погонная масса); $\nu=0,34$ (коэффициент Пуассона); $D=824,476$ (изгибная жесткость); $p^{-}-$ $p^{+}=5000 ; \alpha=0,1 ; \alpha_{*}=0,1$. Все значения приведены в единицах СИ. При реализации метода Галеркина выбрано $n=5$. Начальные условия зададим в виде: $w(x, 0)=0, \dot{w}(x, 0)=0, u(x, 0)=0$, $\dot{u}(x, 0)=0$. Получены графики функций $u(x, t), w(x, t)$ в точке $x=0,8$ (рис. 7 ).

4. Заключение. Построены математические модели системы «трубопровод - датчик давления», и пластины-полосы в сверхзвуковом потоке газа. Рассмотрены линейные и нелинейные модели, описывающие динамику упругого элемента. На основе математической модели системы «трубопровод - датчик давления» методами теории функций комплексного переменного, методом Фурье получено уравнение, связывающее закон изменения рабочей среды на входе в трубопровод (на выходе из камеры сгорания двигателя) и деформацию упругого элемента датчика. В случае математической модели пластины-полосы в сверхзвуковом потоке газа операционным методом получено уравнение, описывающее динамику упругой пластины с учетом аэрогидродинамического воздействия на нее и содержащее лишь функцию деформации. Разработан метод решения полученных уравнений, на основе которого проведен численный эксперимент. В задаче о динамике пластины в сверхзвуковом потоке газа построен функционал, с помощью которого получены аналитические условия устойчивости. Полученные результаты справедливы для нескольких типов закрепления упругого элемента.

\section{СПИСОК ЛИТЕРАТУРЫ}

1. Агеев Р. В., Могилевич Л. И., Попов В. С. Колебания стенок щелевого канала с вязкой жидкостью, образованного трехслойным и твердым дисками// Пробл. машиностр. надежн. машин. - 2014. - 1. - C. 3-11.

2. Агеев Р. В., Могилевич Л. И., Попов В. С., Попова А. А. Движение вязкой жидкости в плоском канале, образованном вибрирующим штампом и шарнирно опертой пластиной// Тр. МАИ. - 2014. 78. - C. $1-13$.

3. Анкилов А. В., Велъмисов П. А. Функционалы Ляпунова в некоторых задачах динамической устойчивости аэроупругих конструкций. - Ульяновск: УлГТУ, 2015.

4. Анкилов А. В., Велъмисов П. А., Горбоконенко В. Д., Покладова Ю. В. Математическое моделирование механической системы «трубопровод-датчик давления». - Ульяновск: УлГТУ, 2008. 
5. Болотин В. В., Радин В. П., Чирков В. П., Щугорев А. В. Устойчивость участка трубопровода с упругой опорой// Изв. РАН. Мех. тв. тела. - 2009. - 1. - С. 174-184.

6. Бочкарев C. A., Матвеенко В. П. Решение задачи о панельном флаттере оболочечных конструкций методом конечных элементов// Мат. модел. - 2002. - 2. - С. 55-71.

7. Вельмисов П. А., Горбоконенко В. Д., Решетников Ю. А. Математическое моделирование механической системы «трубопровод-датчик давления» // Датчики и системы. - 2003. - 6. - С. 12-15.

8. Вельмисов П. А., Манжсосов В. К. Математическое моделирование в задачах динамики виброударных и аэроупругих систем. - Ульяновск: УлГТУ, 2014.

9. Вельмисов П. А., Покладова Ю. В. Исследование динамики деформируемых элементов некоторых аэрогидроупругих систем. - Ульяновск: УлГТУ, 2018.

10. Велъмисов П. А., Покладова Ю. В. Математическое моделирование динамики защитной поверхности резервуара// Вестн. Ульяновск. гос. техн. ун-та. - 2018. - 2. - С. 27-35.

11. Вельмисов П. А., Покладова Ю. В. Математическое моделирование динамики упругих элементов, взаимодействующих с потоком газа// Вестн. Ульяновск. гос. техн. ун-та. - 2018. - 3. - C. 22-30.

12. Вельмисов П. А., Покладова Ю. В., Серебрянникова Е. С. Математическое моделирование системы «трубопровод - датчик давления»// Ж. Средневолж. мат. о-ва. - 2010. - 12, № 4. - С. 85-93.

13. Гахов Ф. Д. Краевые задачи. - М.: Наука, 1977.

14. Зефиров С. В., Кочетков А. В., Овчинников В. Ф., Савихин А. О., Смирнов Л. В., Яскеляин А. В. Численное моделирование динамического деформирования пространственного трубопровода с жидкостью при локальном ударном нагружении // Пробл. прочн. пластичн. - 2013. - 75, № 2. - С. $152-159$.

15. Ильгамов M. А., Хакимов А. Г. Изгиб длинного трубопровода, находящегося в очень вязкой жидкости// Строит. мех. инж. констр. сооруж. - 2009. - 1. - С. 7-12.

16. Казарян А. А., Грошев Г. П. Универсальный датчик давления// Измерит. техн. - 2008. - 3. - С. 2630.

17. Куликов A. Н. Бифуркация автоколебаний при малом коэффициенте демпфирования в сверхзвуковом потоке газа// Прикл. мат. мех. - 2009. - 73, № 2. - С. 271-281.

18. Лаврентъев М. А., Шабат Б. В. Методы теории функций комплексного переменного. - М.: Наука, 1987.

19. Мартыненко В. Т. Исследование причин, влияющих на погрешность преобразования датчиков разности давлений «Сапфир-22»// Приборы и системы. Управление, контроль, диагностика. - 2005. 6. - C. 31-33.

20. Михайлов П. Г., Мокров Е. А., Митрохин С. В., Сергеев Д. А. Особенности метрологического обеспечения современных датчиков пульсаций давлений// Изв. ЮФУ. Техн. науки. - 2012. - 130, № 5. C. $174-179$.

21. Могилевич Л. И., Попов В. С., Попова А. А., Христофорова А. В. Математическое моделирование динамики взаимодействия сильновязкой жидкости со стенками канала, установленного на упругом основании // Динам. сист. мех. машин. - 2016. - 3, № 1. - С. 350-354.

22. Покладова Ю. В., Решетников Ю. А. Математическое моделирование динамики упругого элемента датчика давления в трубопроводе конечной длины// Прикл. мат. мех. (Ульяновск). - 2004. - 6. C. $114-120$.

23. Стучебников B. М. Датчики давления МИДА для систем коммерческого учета энергоносителей// Датчики и системы. - 2009. - 4. - С. 38-40.

24. Эткин Л. Г. Виброчастотные датчики. Теория и практика. - М.: Изд-во МГТУ им. Н. Э. Баумана, 2004.

25. Abdelbaki A. R., Paidoussis M. P., Misra A. K. A nonlinear model for a free-clamped cylinder subjected to confined axial flow// J. Fluids Struct. — 2018. — 80. — P. 390-404.

26. Abdelbaki A. R., Paidoussis M. P., Misra A. K. A nonlinear model for a hanging tubular cantilever simultaneously subjected to internal and confined external axial flows// J. Sound Vibration. — 2019. — 449. — P. 349-367.

27. Faal R. T., Derakhshan D. Flow-induced vibration of pipeline on elastic support// Proc. Eng. — 2011. 14. - P. 2986-2993.

28. Gatica G. N., Heuer N., Meddahi S. Coupling of mixed finite element and stabilized boundary element methods for a fluid-solid interaction problem in 3D// Numer. Methods Partial Differ. Equations. - 2014. — 30, № 4. - P. 1211-1233. 
29. Kontzialis K., Moditis K., Paidoussis M. P. Transient simulations of the fluid-structure interaction response of a partially confined pipe under axial flows in opposite directions// J. Press. Vessel Techn. — 2017. — 139, № 3. - P. 1-8.

30. Moditis K., Paidoussis M., Ratigan J. Dynamics of a partially confined, discharging, cantilever pipe with reverse external flow// J. Fluids Struct. - 2016. - 63. - P. 120-139.

31. Mogilevich L. I., Popova A. A., Popov V. S. On the dynamic interaction of an elastic cylindrical shell with a fluid laminar stream inside in application to pipeline transportation// Sci. Technol. Transport. — 2007. - 2. - P. 69-72.

32. Velmisov P. A., Ankilov A. V. Dynamic stability of plate interacting with viscous fluid// Cybern. Phys. 2017. - 6, № 5. - P. 262-270.

33. Velmisov P. A., Ankilov A. V. Stability of solutions of initial boundary-value problems of aerohydroelasticity// J. Math. Sci. — 2018. — 233, № 6. — P. 958-975.

34. Velmisov P. A., Ankilov A. V., Pokladova Yu. V. Stability of solutions of initial-boundary value problems in aerohydroelasticity// AIP Conf. Proc. - 2018. — 2048, № 1. - 040011.

Вельмисов Петр Александрович

Ульяновский государственный технический университет

E-mail: velmisov@ulstu.ru

Покладова Юлия Валерьевна

Ульяновский государственный технический университет

E-mail: pokladovau@inbox.ru

Мизхер Усама Джавад

Ульяновский государственный технический университет

E-mail: usama.mizher@gmail.com 